\title{
STANLEY DECOMPOSITIONS AND POLARIZATION
}

\author{
SARFRAZ AHMAD
}

\begin{abstract}
We define nice partitions of the multicomplex associated to a Stanley ideal. As the main result we show that if the monomial ideal $I$ is a CM Stanley ideal, then $I^{p}$ is a Stanley ideal as well, where $I^{p}$ is the polarization of $I$.

Key words : Monomial Ideals, Partitionable Simplicial Complexes, Multicomplexes, Stanley Ideals, Polarization.

2010 Mathematics Subject Classification: Primary 13H10, Secondary 13C14, 13F20, $13 F 55$.
\end{abstract}

\section{INTRODUCTION}

Let $K$ be a field and $S=K\left[x_{1}, \ldots, x_{n}\right]$ be a polynomial ring in $n$ variables. Let $I \subset$ $S$ be a monomial ideal, $u \in S / I$ be a monomial and $Z \subseteq\left\{x_{1}, \ldots, x_{n}\right\}$. We denote by $u K[Z]$ the $K$-subspace of $S / I$ generated by all elements $u v$ where $v$ is a monomial in $K[Z]$. The $K$-subspace $u K[Z] \subset S / I$ is called a Stanley space of dimension $|Z|$, if $u K[Z]$ is a free $K[Z]$-module. A decomposition of $S / I$ as a finite direct sum of Stanley spaces $\mathcal{P}: S / I=\oplus_{i=1}^{r} u_{i} K\left[Z_{i}\right]$ is called a Stanley decomposition. Stanley [18 conjectured that there always exists such a decomposition such that $\left|Z_{i}\right| \geq \operatorname{depth}(S / I)$. If Stanley conjecture holds for $S / I$ then $I$ is called a Stanley ideal. The conjecture is still open but true in some special cases [2], [3], 6], [7, [9], [11], [13, [15], [16], [17].

Let $\Gamma$ be a subset of $\mathbb{N}_{\infty}^{n}$. An element $m \in \Gamma$ is called maximal if there is no $a \in \Gamma$ with $a>m$. We denote by $\mathcal{M}(\Gamma)$ the set of maximal elements of $\Gamma$. If $a \in \Gamma$, we call $\operatorname{infpt}(a)=\{i: a(i)=\infty\}$. An element $a \in \Gamma$ is called a facet of $\Gamma$ if for all $m \in \mathcal{M}(\Gamma)$ with $a \leq m$ one has $|\operatorname{infpt}(a)|=|\operatorname{infpt}(m)|$. Herzog and Popescu [10] modify the Stanley's definition of multicomplexes [18]. $\Gamma$ is called a multicomplex if for all $a \in \Gamma$ and for all $b \in \mathbb{N}_{\infty}^{n}$ with $b \leq a$ it follows that $b \in \Gamma$ and for all $a \in \Gamma$ there is a maximal element $m$ in $\Gamma$ such that $a \leq m$. We define an interval $\mathcal{I}$ of $\Gamma$ as a subset of $\Gamma$ for which there exists $a \leq b$ in $\Gamma$ such that $\mathcal{I}=[a, b]=\{c \in \Gamma: a \leq c \leq b\}$. A partition $\mathcal{P}: \Gamma=\bigcup_{i=1}^{t}\left[a_{i}, b_{i}\right]$ of $\Gamma$ is a presentation of $\Gamma$ as a finite disjoint union of intervals $\left[a_{i}, b_{i}\right]$.

Monomial ideals $I$ in the polynomial ring $S=K\left[x_{1}, \ldots, x_{n}\right]$ and multicomplexes in $\mathbb{N}_{\infty}^{n}$ correspond each other bijectively. The multicomplex associated to a monomial ideal $I$ is denoted by $\Gamma(I)$ and similarly, $I(\Gamma)$ denotes the monomial ideal associated to the multicomplex $\Gamma$. We show that Stanley's conjecture holds for $S / I$ if and only if there exists a partition of the multicomplex $\Gamma(I)$ such that $\left|\operatorname{infpt}\left(b_{i}\right)\right| \geq$ 
$\operatorname{depth}(S / I)$ for all $i$. Any partition of a multicomplex satisfying this condition will be called nice.

Let $I \subset S=K\left[x_{1}, \ldots, x_{n}\right]$ be a monomial ideal and $\Gamma(I)$ be the multicomplex associated to $I$. In (Proposition 1.3), we show that a partition $\mathcal{P}: \Gamma(I)=\cup_{i=1}^{t}\left[a_{i}, b_{i}\right]$ of $\Gamma(I)$ is nice if all $b_{i}$ 's are facets of $\Gamma(I)$. Also, when $S / I$ is Cohen-Macaulay, we have this result in both directions (see Corollary 1.4).

Let $I^{p}$ be the polarization of the monomial ideal $I$ and let $\Gamma^{p}$ be the multicomplex associated to $I^{p}$. In Theorem 2.4, we prove that in case of Cohen-Macaulay monomial ideals, if $\Gamma$ has a nice partition then $\Gamma^{p}$ has a nice partition. The converse of this theorem is still open.

Acknowledgements. The author like to thank Professor Jürgen Herzog for his valuable suggestions which improves the final form of the paper.

\section{Partitions of Multicomplexes}

Let $\Gamma$ be a subset of $\mathbb{N}^{n}$. We define on $\mathbb{N}^{n}$ the partial order given by

$$
(a(1), \ldots, a(n)) \leq(b(1), \ldots, b(n))
$$

if $a(i) \leq b(i)$ for all $i$. According to Stanley [18] $\Gamma$ is a multicomplex if for all $a \in \Gamma$ and all $b \in \mathbb{N}^{n}$ with $b \leq a$, it follows that $b \in \Gamma$. The elements of $\Gamma$ are called faces.

Herzog and Popescu [10] modify the Stanley's definition of multicomplexes. Before giving this definition we introduce some notations. We set $\mathbb{N}_{\infty}=\mathbb{N} \cup\{\infty\}$. As usual we set $a \leq \infty$ for all $a \in \mathbb{N}$, and extend the partial order on $\mathbb{N}^{n}$ naturally to $\mathbb{N}_{\infty}^{n}$. Thus now we take $\Gamma$ as a subset of $\mathbb{N}_{\infty}^{n}$.

An element $m \in \Gamma$ is called maximal if there is no $a \in \Gamma$ with $a>m$. We denote by $\mathcal{M}(\Gamma)$ the set of maximal elements of $\Gamma$. If $a \in \Gamma$, we call

$$
\operatorname{infpt}(a)=\{i: a(i)=\infty\}
$$

the infinite part of $a$.

Definition 1.1. A subset $\Gamma \subset \mathbb{N}_{\infty}^{n}$ is called a multicomplex if

(1) for all $a \in \Gamma$ and for all $b \in \mathbb{N}_{\infty}^{n}$ with $b \leq a$ it follows that $b \in \Gamma$,

(2) for all $a \in \Gamma$ there exists an element $m \in \mathcal{M}(\Gamma)$ such that $a \leq m$.

An element $a \in \Gamma$ is called a facet of $\Gamma$ if for all $m \in \mathcal{M}(\Gamma)$ with $a \leq m$ one has $\operatorname{infpt}(a)=\operatorname{infpt}(m)$. The set of all facets of $\Gamma$ will be denoted by $\mathcal{F}(\Gamma)$. In [10] it is shown that each multicomplex has only a finite number of facets.

Monomial ideals $I$ in the polynomial ring $S=K\left[x_{1}, \ldots, x_{n}\right]$ and multicomplexes in $\mathbb{N}_{\infty}^{n}$ correspond each other bijectively. The bijection is defined as follows: Let $\Gamma$ be a multicomplex, and let $I(\Gamma)$ be the $K$-subspace in $S=K\left[x_{1}, \ldots, x_{n}\right]$ spanned by all monomials $x^{a}$ such that $a \notin \Gamma$. Note that if $a \in \mathbb{N}_{\infty}^{n}$ and $b \in \mathbb{N}_{\infty}^{n} \backslash \Gamma$, then $a+b \in \mathbb{N}_{\infty}^{n} \backslash \Gamma$, that is, if $x^{b} \in I(\Gamma)$ then $x^{a} x^{b} \in I(\Gamma)$ for all $x^{a} \in S$. In other words, $I(\Gamma)$ is a monomial ideal. In particular, the monomials $x^{a}$ with $a \in \Gamma$ form a $K$-basis of $S / I(\Gamma)$.

Conversely, given an arbitrary monomial ideal $I \subset S$, there is a unique multicomplex $\Gamma$ with $I=I(\Gamma)$, namely the smallest multicomplex (with respect to inclusion) 
which contains $A=\left\{a \in \mathbb{N}_{\infty}^{n}: x^{a} \notin I\right\}$. Such a multicomplex exists and is uniquely determined since an arbitrary intersection of multicomplexes is again a multicomplex.

One has the following obvious rules: let $\left\{\Gamma_{j}, j \in J\right\}$ be a family of multicomplexes. Then

(a) $I\left(\bigcap_{j \in J} \Gamma_{j}\right)=\sum_{j \in J} I\left(\Gamma_{j}\right)$,

(b) if $J$ is finite, then $I\left(\bigcup_{j \in J} \Gamma_{j}\right)=\bigcap_{j \in J} I\left(\Gamma_{j}\right)$

Let $\Gamma \subset \mathbb{N}_{\infty}^{n}$ be a multicomplex. We define an interval $\mathcal{I}$ of $\Gamma$ as a subset of $\Gamma$ for which there exists $a \leq b$ in $\Gamma$ such that $\mathcal{I}=\{c \in \Gamma: a \leq c \leq b\}$. We denote an interval given by faces $a$ and $b$ as $[a, b]$. A partition $\mathcal{P}$ of $\Gamma$ is a presentation of $\Gamma$ as a finite disjoint union of intervals.

Lemma 1.2. Let $\mathcal{P}: \Gamma=\bigcup_{i=1}^{t}\left[a_{i}, b_{i}\right]$ be a partition of $\Gamma$. Then $\operatorname{infpt}\left(a_{i}\right)=\emptyset$ for all $i$.

Proof. Assume that for some $i$, say for $i=1$, we have $\operatorname{infpt}\left(a_{1}\right) \neq \emptyset$. We may assume that $a_{1}(1)=\infty$. Set $a=a_{1}$ and let $c$ be any integer. None of the faces $(c, a(2), \ldots, a(n))$ belong to $\left[a_{1}, b_{1}\right]$. Thus for each $c$ there exist an $i \in\{2, \ldots, t\}$ such that $(c, a(2), \ldots, a(n)) \in\left[a_{i}, b_{i}\right]$. Hence for some $j>1$, infinitely of the vectors $(c, a(2), \ldots, a(n))$ belong to $\left[a_{j}, b_{j}\right]$. This is only possible if $(\infty, a(2), \ldots, a(n))$ belongs to $\left[a_{j}, b_{j}\right]$. This is a contradiction, since $a_{1}=(\infty, a(2), \ldots, a(n)) \in\left[a_{1}, b_{1}\right]$.

Let $I \subset S=K\left[x_{1}, \ldots, x_{n}\right]$ be a monomial ideal. Any decomposition of $S / I$ as a direct sum of $K$-vector spaces of the form $u K[Z]$, where $u$ is a monomial in $K[X]$ and $Z \subset X=\left\{x_{1}, \ldots, x_{n}\right\}$, is called a Stanley decomposition if $u K[Z]$ is a free $K[Z]$-module. In this paper we will call $u K[Z]$ a Stanley space of dimension $|Z|$, where $|Z|$ denotes the cardinality of $Z$. Stanley decomposition have been studied in various combinatorial and algebraic contexts, see [2], [3], [6], [7], [9], [11], [13], [15], [16], [17].

Stanley [18] conjectured that there always exists a Stanley decomposition

$$
S / I=\bigoplus_{i=1}^{r} u_{i} K\left[Z_{i}\right],
$$

such that $\left|Z_{i}\right| \geq \operatorname{depth}(S / I)$ for all $i$.

Next we describe how Stanley decompositions and partitions are related to each other. Let $\Gamma \subset \mathbb{N}_{\infty}^{n}$ be a multicomplex, $[a, b] \subset \Gamma$ an interval and $U_{[a, b]}$ the $K$-subspace of $S$ generated by all monomials $u=x_{1}^{c(1)} \cdots x_{n}^{c(n)}$ such that $c=$ $(c(1), \ldots, c(n)) \in[a, b]$. Then obviously one has that $U_{[a, b]}$ is a Stanley space if and only if

(i) $\operatorname{infpt}(a)=\emptyset$,

(ii) $i \notin \operatorname{infpt}(b) \Rightarrow a(i)=b(i)$.

Indeed in this case $U_{[a, b]}=x^{a}\left[Z_{b}\right]$, where $Z_{b}=\left\{x_{i}: b(i)=\infty\right\}$.

Let $I \subset S$ be a monomial ideal and $\Gamma(I)$ be the multicomplex associated to $I$. Also 
let $S / I=\oplus_{i=1}^{r} x^{a_{i}} K\left[Z_{i}\right]$ be a Stanley decomposition of $S / I$. Set $b_{i}(j)=\infty$ if $x_{j} \in Z_{i}$ and $b_{i}(j)=a_{i}(j)$ if $x_{j} \notin Z_{i}$. Then $\cup_{i=1}^{r}\left[a_{i}, b_{i}\right]$ is a partition of $\Gamma(I)$. For instance, if $a \in\left[a_{i}, b_{i}\right] \cap\left[a_{j}, b_{j}\right] \cap \mathbb{N}^{n}$ for $i, j \in\{1, \ldots, r\}$ and $i \neq j$, then $x^{a} \in a_{i} K\left[Z_{i}\right] \cup a_{j} K\left[Z_{j}\right]$, a contradiction. Thus $\cup_{i=1}^{r}\left[a_{i}, b_{i}\right]$ is disjoint.

Conversely, we observe that each interval $[a, b]$ with $\operatorname{infpt}(a)=\emptyset$ can be written as disjoint union of intervals

$$
[a, b]=\cup\left[c_{i}, b_{i}\right]
$$

such that each $\left[c_{i}, b_{i}\right]$ corresponds to a Stanley space. Indeed, if, as we may assume, for some integer $r$ we have that $b(k)<\infty$ for $k \leq r$ and $b(k)=\infty$ for $k>r$. Then $[a, b]$ is the disjoint union of the intervals

$$
[(c(1), \ldots, c(r), a(r+1), \ldots, a(n)),(c(1), \ldots, c(r), \infty, \ldots, \infty)]
$$

with $a(k) \leq c(k) \leq b(k)$ for $k=1, \ldots, r$, and each of these intervals satisfies (i) and (ii). Therefore, due to (11) and Lemma 1.2, Stanley's conjecture holds for $S / I$ if and only if there exists a partition $\mathcal{P}: \Gamma=\bigcup_{i=1}^{t}\left[a_{i}, b_{i}\right]$ of the multicomplex $\Gamma=\Gamma(I)$ such that

$$
\left|\operatorname{infpt}\left(b_{i}\right)\right| \geq \operatorname{depth}(S / I(\Gamma)) \text { for all } i \text {. }
$$

Any partition of a multicomplex satisfying condition (2) will be called nice.

Proposition 1.3. A partition $\mathcal{P}: \Gamma=\bigcup_{i=1}^{t}\left[a_{i}, b_{i}\right]$ of the multicomplex $\Gamma$ is a nice partition if $b_{i} \in \mathcal{F}(\Gamma)$ for all $i$.

Proof. Let $I(\Gamma)=\bigcap_{i=1}^{m} Q_{i}$ be the unique irredundant presentation of $I$ as an intersection irreducible monomial ideals, and let $P_{i}=\sqrt{Q_{i}}$ for $i=1, \ldots, m$. Then $\operatorname{Ass}(S / I)=\left\{P_{1}, \ldots, P_{m}\right\}$.

By [10, Proposition 9.12] there is a bijection between the $Q_{i}$ and the set of $\mathcal{M}(\Gamma)$ of maximal faces of $\Gamma$. In fact, for each $i$ there is a unique $m_{i} \in \mathcal{M}(\Gamma)$ such that $Q_{i}=I\left(\Gamma\left(m_{i}\right)\right)$ where $\Gamma\left(m_{i}\right)$ denotes the smallest multicomplex containing $m_{i}$. The assignment $Q_{i} \mapsto m_{i}$ establishes this bijection. Moreover, $\operatorname{dim} S / P_{i}=\operatorname{infpt}\left(m_{i}\right)$ for all $i$. Therefore,

$$
\begin{aligned}
\min \left\{\left|\operatorname{infpt}\left(b_{i}\right)\right|: b_{i} \in \mathcal{F}(\Gamma)\right\} & =\min \left\{\left|\operatorname{infpt}\left(m_{j}\right)\right|: m_{j} \in \mathcal{M}(\Gamma)\right\} \\
& =\min \left\{\operatorname{dim}\left(S / P_{j}\right): P_{j} \in \operatorname{Ass}(S / I(\Gamma))\right\} \\
& \geq \operatorname{depth}(S / I(\Gamma)) .
\end{aligned}
$$

The first equation follows from the definition of the facets, while the last inequality is a basic fact of commutative algebra, see [5, Proposition 1.2.13]. These considerations show that our given partition is nice.

Corollary 1.4. Let $I \subset S$ be a monomial ideal such that $S / I$ is Cohen-Macaulay. Let $\Gamma$ be the multicomplex associated to $I$ and $\mathcal{P}: \Gamma=\bigcup_{i=1}^{t}\left[a_{i}, b_{i}\right]$ be a partition of $\Gamma$. Then the following conditions are equivalent.

(a) $\mathcal{P}$ is nice.

(b) $\left\{b_{1}, \ldots, b_{t}\right\} \subseteq \mathcal{F}(\Gamma)$ 
(c) $\mathcal{M}(\Gamma) \subseteq\left\{b_{1}, \ldots, b_{t}\right\} \subseteq \mathcal{F}(\Gamma)$

Proof. (a) $\Rightarrow$ (b): In case $S / I$ is Cohen-Macaulay we have $|\operatorname{infpt}(b)| \leq \operatorname{depth}(S / I)$ for all faces of $\Gamma$, and equality holds for $b$ if and only if $b$ is a facet. Thus $\mathcal{P}$ can be nice only if $\left\{b_{1}, \ldots, b_{t}\right\} \subseteq \mathcal{F}(\Gamma)$.

(b) $\Rightarrow$ (c): Let $m \in \mathcal{M}(\Gamma)$; then $m \in\left[a_{i}, b_{i}\right]$ for some $i$. Since $m \leq b_{i}$ and since $m$ is maximal it follows that $m=b_{i}$. Thus $\mathcal{M}(\Gamma) \subseteq\left\{b_{1}, \ldots, b_{t}\right\}$.

(c) $\Rightarrow$ (a) follows from Proposition 1.3.

Remark 1.5. In the above Corollary if $\mathcal{P}$ is nice then we can refine it in such a way that for the refinement

$$
\mathcal{P}^{\prime}: \Gamma=\bigcup_{i=1}^{t^{\prime}}\left[a_{i}^{\prime}, b_{i}^{\prime}\right]
$$

we have $\left\{b_{1}^{\prime}, \ldots, b_{t^{\prime}}^{\prime}\right\}=\mathcal{F}(\Gamma)$. To prove this fact we first observe that $\left|\operatorname{infpt}\left(a_{i}\right)\right|=0$ for all $i$, see Lemma 1.2. Since $\mathcal{F}(\Gamma)=\bigcup_{i=1}^{t}\left(\mathcal{F}(\Gamma) \cap\left[a_{i}, b_{i}\right]\right)$, it is enough to write each interval $\left[a_{i}, b_{i}\right]$ as a disjoint union of intervals $\bigcup_{j=1}^{l}\left[c_{j}, e_{j}\right]$ where $\left\{e_{1}, e_{2}, \ldots, e_{l}\right\}=$ $\mathcal{F}(\Gamma) \cap\left[a_{i}, b_{i}\right]$

For simplicity, we may assume that $b_{i}(k)<\infty$ for $k \leq r$ and $b_{i}(k)=\infty$ for $k>r$. Then $e \in\left[a_{i}, b_{i}\right]$ is a facet of $\Gamma$ if and only if $a_{i}(k) \leq e(k) \leq b_{i}(k)$ for $k \leq r$ and $e(k)=\infty$ for $k>r$. Thus if we set $c_{j}(k)=e_{j}(k)$ for $k \leq r$ and $c_{j}(k)=a_{i}(k)$ for $k>r$, then $\left[a_{i}, b_{i}\right]=\bigcup_{j=1}^{l}\left[c_{j}, e_{j}\right]$ is the desired refinement of $\left[a_{i}, b_{i}\right]$.

\section{Partitions and Polarization}

Let $S=K\left[x_{1}, \ldots, x_{n}\right]$ be the polynomial ring in $n$ variables over the field $K$, and $u=\prod_{i=1}^{n} x_{i}^{a_{i}}$ be the monomial in $S$. Then

$$
u^{p}=\prod_{i=1}^{n} \prod_{j=1}^{a_{i}} x_{i j} \in K\left[x_{11}, \ldots, x_{1 a_{1}}, \ldots, x_{n 1}, \ldots, x_{n a_{n}}\right]
$$

is called the polarization of $u$.

Let $I$ be the monomial ideal in $S$ with monomial generators $u_{1}, \ldots, u_{r}$. Then $\left(u_{1}^{p}, \ldots, u_{r}^{p}\right)$ is called a polarization of $I$ and is denoted by $I^{p}$. It is known that $I$ is Cohen-Macaulay if and only if $I^{p}$ is Cohen-Macaulay. Indeed, the elements $x_{i j}-x_{i 1}$, $i=1, \ldots, n$ and $j=1,2, \ldots$ form a regular sequence on $T / I^{p}$, and $T / I^{p}$ modulo this regular sequence is isomorphic to $S / I$.

Let $I=\left(u_{1}, \ldots, u_{s}\right) \subset S$ be a monomial ideal. We may assume that for each $i \in[n]$ there exists $j$ such that $x_{i}$ divides $u_{j}$. Let $u_{j}=x_{1}^{a_{j 1}} \cdots x_{n}^{a_{j n}}$ for $j=1, \ldots, s$ and set $r_{i}=\max a_{j i}: j=1, \ldots, s$ for $i=1, \ldots, n$. Moreover we set $r=\sum_{i=1}^{n} r_{i}$.

Let $I=\bigcap_{i=1}^{t} Q_{i}$ be the unique irredundant presentation of $I$ as an intersection of irreducible monomial ideals. In particular, each $Q_{i}$ is generated by pure powers of some of the variables. Then $I^{p}=\bigcap_{i=1}^{t_{1}} Q_{i}^{p}$ is an ideal in the polynomial ring

$$
T=K\left[x_{11}, \ldots, x_{1 r_{1}}, x_{21}, \ldots, x_{n 1}, \ldots, x_{n r_{n}}\right]
$$

in $r$ variables. 
We denote by $\Gamma, \Gamma^{p}, \Gamma_{i}$ and $\Gamma_{i}^{p}$ the multicomplexes associated to $I, I^{p}, Q_{i}$ and $Q_{i}^{p}$, respectively, and by $\mathcal{F}, \mathcal{F}^{p}, \mathcal{F}_{i}$ and $\mathcal{F}_{i}^{p}$ the set of facets of $\Gamma, \Gamma^{p}, \Gamma_{i}$ and $\Gamma_{i}^{p}$, respectively.

Each $\Gamma_{i}$ has only one maximal facet, say $m_{i}$, and $m_{i}(k) \leq r_{k}-1$ for all $k$ with $m_{i}(k) \neq \infty$. Moreover, $\mathcal{M}(\Gamma)=\left\{m_{1}, \ldots, m_{t}\right\}$. It follows that the set of facets of $\Gamma$ is a subset of the set

$$
\mathcal{B}=\left\{b \in \mathbb{N}_{\infty}^{n}: b(i)<r_{i} \text { if } b(i) \neq \infty\right\} .
$$

We define the map

$$
\beta: \mathcal{B} \rightarrow\{0, \infty\}^{r}, \quad b \mapsto b^{\prime},
$$

where the components of the vectors $b^{\prime}$ are indexed by pairs of numbers $i j$, where for each $i=1, \ldots, n$ the second index $j$ runs in the range $j=1, \ldots, r_{i}$. The map $\beta$ is defined as follows:

$$
b^{\prime}(i j)= \begin{cases}0, & \text { if } b(i)<\infty \text { and } j=b(i)+1, \\ \infty, & \text { otherwise }\end{cases}
$$

We quote the following result by Soleyman Jahan [12, Proposition 3.8]

Proposition 2.1. With the above assumptions and notation the restriction of the map $\beta$ to $\mathcal{F}$ induces a bijection $\mathcal{F} \rightarrow \mathcal{F}^{p}$.

The following example demonstrates this bijection: let $I=\left(x_{1}^{2}, x_{1} x_{2}, x_{3}^{2}\right)=$ $\left(x_{1}, x_{3}^{2}\right) \cap\left(x_{1}^{2}, x_{2}, x_{3}^{2}\right) \subset K\left[x_{1}, x_{2}, x_{3}\right]$. Then the multicomplex $\Gamma$ associated to $I$ has facets

$$
(0, \infty, 0),(0, \infty, 1),(1,0,0),(1,0,1),
$$

while the multicomplex of the polarized ideal

$$
I^{p}=\left(x_{11} x_{12}, x_{11} x_{21}, x_{31} x_{32}\right) \subset K\left[x_{11}, x_{12}, x_{21}, x_{31}, x_{32}\right] .
$$

has the facets

$$
(0, \infty, \infty, 0, \infty),(0, \infty, \infty, \infty, 0),(\infty, 0,0,0, \infty),(\infty, 0,0, \infty, 0) .
$$

Let $\Gamma=\bigcup_{i=1}^{t}\left[a_{i}, b_{i}\right]$ be a nice partition of $\Gamma$ with $\mathcal{F}(\Gamma)=\left\{b_{1}, \ldots, b_{t}\right\}$. With the notation introduced above we have

Lemma 2.2. $a_{i}(j) \leq r_{j}$ for all $i$ and $j$.

Proof. Suppose without loss of generality that $a_{1}(1)>r_{1}$. Then $b_{1}(1)=\infty$, because if $b_{1}(1)<\infty$ it follows that $a_{1}(1) \leq b_{1}(1)<r_{1}$, a contradiction. Now since $\Gamma=$ $\bigcup_{i=1}^{t}\left[a_{i}, b_{i}\right]$ and since $a=\left(r_{1}, a_{1}(2), \ldots, a_{1}(n)\right) \in \Gamma \backslash\left[a_{1}, b_{1}\right]$, there exists $i>1$ such that $a \in\left[a_{i}, b_{i}\right]$. As above $b_{i}(1)=\infty$ because if $b_{i}(a)<\infty$ then $r_{1} \leq b_{i}(1)<r_{1}$, which is not possible. Hence we conclude that $a_{i} \leq a<a_{1}<b_{i} \Rightarrow a_{1} \in\left[a_{i}, b_{i}\right]$, a contradiction.

We want to "polarize" the nice partition $\Gamma=\bigcup_{i=1}^{t}\left[a_{i}, b_{i}\right]$. For this purpose we consider the set $\mathcal{A}=\left\{a \in \mathbb{N}: a(i) \leq r_{i}\right\}$ and the following map $\gamma: \mathcal{A} \rightarrow\{0,1\}^{r}$ with

$$
\gamma(a)(i j)=\left\{\begin{aligned}
0, & \text { if } j>a(i) \\
1, & \text { otherwise } \\
6 &
\end{aligned}\right.
$$


We observe that $\gamma$ is injective. Indeed, for $a \neq a^{\prime}$ there exists $i$ such that $a(i) \neq a^{\prime}(i)$, say, $a(i)<a^{\prime}(i)$. Then $a(i j)=0$ for $j=a(i)+1$, while $a^{\prime}(i j)=1$ for $j=a(i)+1$.

Let $\mathcal{I}=[a, b] \subset \Gamma \subset \mathbb{N}_{\infty}^{n}$ be an interval such that $a=(a(1), a(2), \ldots, a(n))$ and $b=(b(1), b(2), \ldots, b(n))$. We define an $i$-subinterval as

$$
\left\{c \in \mathbb{N}_{\infty}: a(i) \leq c \leq b(i)\right\}
$$

and denote it by $\mathcal{I}(i)=[a(i), b(i)]$.

Example 2.3. Let $a, b \in \Gamma \subset \mathbb{N}_{\infty}^{2} a=(2,5), b=(4, \infty)$, then

$\mathcal{I}(1)=[a(1), b(1)]=[2,4]$ i.e. $\mathcal{I}(1)=\{2,3,4\}$,

$\mathcal{I}(2)=[a(2), b(2)]=[5, \infty]$ i.e. $\mathcal{I}(2)=\{5,6, \ldots\}$.

Next we need the following elementary lemma.

Lemma 2.4. Let $\mathcal{I}_{1}, \mathcal{I}_{2}$ be two intervals of a multicomplex $\Gamma \subset \mathbb{N}_{\infty}^{n}$ such that $\mathcal{I}_{1}=$ $[a, b]$ and $\mathcal{I}_{2}=[c, d]$. Suppose $\mathcal{I}_{1} \cap \mathcal{I}_{2}=\emptyset$, then there exists $i$ such that $\mathcal{I}_{1}(i) \cap \mathcal{I}_{2}(i)=$ $\emptyset$.

Let $I \subset S$ be a monomial ideal and let $S / I=\bigoplus_{i=1}^{r} u_{i} K\left[Z_{i}\right]$ be its Stanley decomposition, where $u_{i}=x^{a_{i}}$ for $i=1, \ldots, r$. Then the Hilbert series is given by $H(S / I)=\sum_{i=1}^{r} t^{\left|a_{i}\right|} /(1-t)^{\left|Z_{i}\right|}$, where $\left|a_{i}\right|$ denotes the sum of the components of $a_{i}$ and $\left|Z_{i}\right|$ the cardinality of $Z_{i}$. Thus if $\Gamma$ is the multicomplex associated to $I$ and $\Gamma=\bigcup_{i=1}^{t}\left[a_{i}, b_{i}\right]$ the corresponding partition (with $b_{i}(j)=a_{i}(j)$ for $x_{j} \notin Z_{i}$ and $b_{i}(j)=\infty$ for $\left.x_{j} \in Z_{i}\right)$, then $H(S / I)=\sum_{i=1}^{r} t^{\left|a_{i}\right|} /(1-t)^{\left|b_{i}\right|_{\infty}}$, where $\left|b_{i}\right|_{\infty}=\left|\operatorname{infpt} b_{i}\right|$.

Theorem 2.5. Let $I \subset S$ be a monomial ideal such that $S / I$ is Cohen-Macaulay, and $I^{p}$ be the polarization of I. Suppose I satisfies the Stanley Conjecture, then $I^{p}$ satisfies it too.

Proof. Let $\Gamma$ be the multicomplex associated to $I$. Since $I$ satisfies the Stanley Conjecture, $\Gamma$ has a nice partition. Let $\Gamma^{p}$ be the multicomplex associated to $I^{p}$. Then we show that $\Gamma^{p}$ has a nice partition.

Let $\Gamma=\bigcup_{i=1}^{\hat{t}}\left[\hat{a}_{i}, \hat{b}_{i}\right]$ be a nice partition of $\Gamma$ then by Corollary $1.4, \hat{b}_{i} \in \mathcal{F}(\Gamma)$ for all $i$. Again by Remark 1.5, we can refine this partition to another nice partition say $\mathcal{P}: \Gamma=\bigcup_{i=1}^{t}\left[a_{i}, b_{i}\right]$ such that $\left\{b_{1}, \ldots, b_{t}\right\}=\mathcal{F}(\Gamma)$.

Let $\beta$ and $\gamma$ be the functions defined above and set $\beta\left(b_{i}\right)=\bar{b}_{i}$ and $\gamma\left(a_{i}\right)=\bar{a}_{i}$ for all $i=1, \ldots, t^{\prime}$. We will show that $\mathcal{P}^{p}: \Gamma^{p}=\bigcup_{i=1}^{t}\left[\bar{a}_{i}, \bar{b}_{i}\right]$ is a nice partition of $\Gamma^{p}$.

$\mathcal{P}^{p}$ is a partition if the intervals $\left[\bar{a}_{i}, \bar{b}_{i}\right]$ are disjoint for all $i=1, \ldots, t$ and $\mathcal{P}^{p}$ covers all the faces of $\Gamma^{p}$.

Suppose that the intervals are not disjoint and say there exist a face $a \in\left[\bar{a}_{i}, \bar{b}_{i}\right] \cap$ $\left[\bar{a}_{j}, \bar{b}_{j}\right]$ for some $i \neq j, i, j \in\{1, \ldots, t\}$. Since $a_{i} \neq a_{j}$ we get $\bar{a}_{i} \neq \bar{a}_{j}, \gamma$ being injective.

The intervals $\left[a_{i}, b_{i}\right]$ and $\left[a_{j}, b_{j}\right]$ are disjoint and so by Lemma 2.4 , there exists at least one pair of $i_{1}$-subintervals say $\left[a_{i}\left(i_{1}\right), b_{i}\left(i_{1}\right)\right]$ and $\left[a_{j}\left(i_{1}\right), b_{j}\left(i_{1}\right)\right]$ for $i_{1} \in\{1, \ldots, n\}$ such that $\left[a_{i}\left(i_{1}\right), b_{i}\left(i_{1}\right)\right] \cap\left[a_{j}\left(i_{1}\right), b_{j}\left(i_{1}\right)\right]=\emptyset$. 
So at least one of $b_{i}\left(i_{1}\right), b_{j}\left(i_{1}\right)$ is finite say $b_{i}\left(i_{1}\right) \neq \infty$, and so by condition $(i i)$ on page $4, b_{i}\left(i_{1}\right)=a_{i}\left(i_{1}\right)$. Also we can assume that $a_{i}\left(i_{1}\right)<a_{j}\left(i_{1}\right)$. If not and $b_{j}\left(i_{1}\right)=\infty$ then $\left[a_{i}\left(i_{1}\right), b_{i}\left(i_{1}\right)\right] \subset\left[a_{j}\left(i_{1}\right), b_{j}\left(i_{1}\right)\right]$ which is not possible, if $b_{j}\left(i_{1}\right)<\infty$ then change $i$ by $j$.

Let $a_{i}\left(i_{1}\right)=b_{i}\left(i_{1}\right)=k$ and $a_{j}\left(i_{1}\right)=m>k$. Then by definition of $\gamma$ and $\beta$ we have $\bar{a}_{i}\left(i_{1} \overline{k+1}\right)=0=\bar{b}_{i}\left(i_{1} \overline{k+1}\right)$ and $\bar{a}_{j}\left(i_{1} l\right)=1$ for $l \leq m$, thus $\bar{a}_{j}\left(i_{1} \overline{k+1}\right)=1$.

It follows $a\left(i_{1} \overline{k+1}\right)=0$. On the other hand since $a \geq \bar{a}_{j}$ we get $a\left(i_{1} \overline{k+1}\right) \geq$ $\bar{a}_{j}\left(i_{1} \overline{k+1}\right)=1$. A contradiction.

Now for the second part of the proof, we will use the Hilbert series. We have $H(S / I)=\sum_{i=1}^{t} s^{\left|a_{i}\right|} /(1-s)^{\left|b_{i}\right|_{\infty}}$. The definition of the function $\gamma$ implies that $\left|a_{i}\right|=\left|\bar{a}_{i}\right|$ for all $i=\{1, \ldots, t\}$. Now for each polarization step, the depth of $S / I$ increases by 1 . Also by definition of $\beta$ for each polarization step, number of infinite points increases by 1 . Thus after $n_{1}$ polarization steps $\left|\operatorname{infpt}\left(\bar{b}_{i}\right)\right|=\left|\operatorname{infpt}\left(b_{i}\right)\right|+n_{1}$. So

$$
H\left(\bigcup_{i=1}^{t}\left[\bar{a}_{i}, \bar{b}_{i}\right]\right)=\sum_{i=1}^{t} \frac{s^{\left|a_{i}\right|}}{(1-s)^{\left|b_{i}\right|_{\infty}+n_{1}}}=\frac{1}{(1-s)^{n_{1}}} H(S / I)
$$

is in fact the Hilbert series of $H\left(S^{p} / I^{p}\right)$. Hence $S^{p} / I^{p}=\cup_{i=1}^{t}\left[\bar{a}_{i}, \bar{b}_{i}\right]$.

Note that $\mathcal{P}^{p}$ is a nice partition because $\left|\bar{b}_{i}\right|_{\infty}=\left|b_{i}\right|_{\infty}+n_{1} \geq \operatorname{depth}_{S}(S / I)+n_{1}=$ $\operatorname{depth}_{S^{p}}\left(S^{p} / I^{p}\right)$ for all $i$.

The converse of above theorem is still open. If one can proof the converse, then the Stanley Conjecture will reduce to the case of squarefree monomial ideals $I$ where $I$ is Cohen Macaulay.

\section{REFERENCES}

[1] S. Ahmad, D. Popescu, Sequentially Cohen-Macaulay monomial ideals of embedding dimension four, Bull. Math. Soc. Sc. Math. Roumanie, 50(98), no 2, (2007), 99-110 (see www.rms.unibuc.ro/bulletin)

[2] I. Anwar, Janet's algorithm, Bull. Math. Soc. Sc. Math. Roumanie 5(99), no. 1 (2008), 11-19.

[3] I. Anwar and D. Popescu, Stanley conjecture in small embedding dimension, J. Alg. 318(2007), 1027-1031.

[4] J. Apel, On a conjecture of R. P. Stanley; Part I-Monomial Ideals, J. of Alg. Comb. 17, (2003), 36-59.

[5] W. Bruns, J. Herzog, Cohen-Macaulay rings, Revised Edition, Cambridge, 1996.

[6] M. Cimpoeas, Stanley depth for monomial complete intersection, Bull. Math. Soc. Sc. Math. Roumanie 51(99), no.3 (2008), 205-211.

[7] M. Cimpoeas, Stanley depth for monomial ideals in three variables, Preprint (2008), Arxiv:Math.AC/0807.2166.

[8] A. Dress, A new algebraic criterion for shellability, Beitr. Algebr. Geom. 340(1), (1993), 45-55.

[9] J. Herzog, A. Soleyman Jahan, S. Yassemi, Stanley decompositions and partitionable simplicial complexes, J. Algebraic Combinatorics, 27 (2008), 113-125.

[10] J. Herzog, D. Popescu, Finite filtrations of modules and shellable multicomplexes, Manuscripta Math. 121, (2006), 385-410.

[11] J. Herzog, M. Vladoiu and X. Zheng, How to compute the Stanley depth of a monomial ideal, J. Algebra, (2009).

[12] A. Soleyman Jahan, Prime filtrations of monomial ideals and polarizations, J. Algebra, 312(2007), 1011-1032. 
[13] S. Nasir, Stanley decomposition and localization, Bull. Math. Soc. Sc. Math. Roumanie 51(99), no.2(2008), 151-158.

[14] D. Popescu, Criterions for shellable multicomplexes, An. St. Univ. Ovidius, Constanta 14(2), (2006),73-84, Arxiv:math.AC/0505655

[15] D. Popescu, Stanley depth of Multigraded modules, J. Algebra 321(2009), 2782-2797.

[16] D. Popescu, Muhammad I. Qureshi, Computing the Stanley depth, Arxiv:Math. $\mathrm{AC} / 0907.0912$

[17] A. Rauf, Stanley Decomposition, Pretty Clean Filtrations and Reductions Modulo Regular Elements, Bull. Math. Soc. Sc. Math. Roumanie 50(98), no4 (2007), 347-354.

[18] R. P. Stanley, Linear Diophantine equations and local cohomology, Invent. Math.68, (1982), 175-193.

[19] R. H. Villarreal, Monomial algebras, Dekker, New York, 2001.

Sarfraz Ahmad, COMSATS Institute of Information Technology, Lahore,Pakistan. E-mail address: sarfraz11@gmail.com 